\title{
Recent experiments in the EAST and HT-7 superconducting tokamaks
}

Baonian Wan 1), Jiangang Li 1), Xianzhu Gong 1), Bingjia Xiao 1), Yuntao Song 1), Yu Wu 1), Jiafang Shan 1), Yanping Zhao 1), Liqun $\mathrm{Hu}$ 1) for the EAST and HT-7 teams, and international collaborators: Paul Anderson 2), Vincent Chan 2), Dave Humphreys 2), Al Hyatt 2), Lang L. Lao 2), Jim Leuer 2), Ali Mahdavi 2), Gary Jackson 2), Ben Penaflor 2), David Piglowski 2), Michael Schaffe 2), Mike Walker 2), He Huang 3), Kenneth Gentle 3), Perry Philippe 3), William Rowan 3), D.Mueller 4), M.Bitter 4), K.W.Hill 4), Houyang Guo 5), R.Kumazawa 6), T.Mutoh 6), K.Saito 6), T.Watari 6), B.Bremond 7), K.Vueillie 7), S.G.Lee 8), J.G.Bak 8)

1) Institute of Plasma Physics, Chinese Academy of Sciences, Hefei, China

2) General Atomic, San Diego, USA

3) Fusion Research Center, UT at Austin, USA

4) Princeton Plasma Physics Laboratory

5) Washington University, Washington, USA

6) National Institute for Fusion Sciences, Toki, Japan

7) Association Euratom-CEA, CEA Cadarache, France

8) NFRC, Korea Basic Science Institute, Daejeon, Korea

e-mail contact of main author: bnwan@ipp.ac.cn

\begin{abstract}
First diverter plasma configuration in EAST was obtained in the second campaign after the last IAEA meeting. To support the long pulse diverted plasma discharges, new capabilities including the fully actively water cooled in-vessel components, current drive and heating power, diagnostics, real-time plasma control algorithm were developed. Pre-program shape and RZIP feedback control produce a variety of shaped plasma, which cover almost designed configuration. RTEFIT/ISOFLUX control algorithm was primarily realized. A number of operational issues, such as plasma initiation, ramp up and configuration control with constraints of superconducting coils were successfully investigated. The physical engineering capability on the superconducting magnetic system of EAST was assessed by simulating discharges. Since the last IAEA meeting, experiments in HT-7 focused on long pulse discharges under different scenarios and high power heating to support EAST experiments both physically and technically. The long pulse discharges up to 400s renews the records in HT-7. The high performance regimes have been greatly extended. The investigation of sawtooth activities in ohmic and LHCD plasmas of HT-7 by 2D ECE image and high resolution soft-X arrays support the turbulence model instead of the fast reconnection of the $m=1$ magnetic island. Coexistence of electron mode and ion mode in high density ohmic plasma has been observed by 2D ECEI in HT-7. The spectral characteristics of GAM and the nonlinear three wave coupling at the GAM frequency has been investigated.
\end{abstract}

\section{Introduction}

Achievement of the first plasma has demonstrated that EAST (Experimental Advanced Superconducting Tokamak) engineering construction is completely successful[1,2]. First diverter plasma configuration in EAST was obtained in the second campaign just after the last IAEA meeting. Fully superconducting coils and strongly shaped plasma configuration are required for future tokamak fusion power plants. EAST is at the moment the only such tokamak in the world, which can address both issues simultaneously. 
EAST is presently equipped with actively cooled plasma facing components (PFCs) and with $8 \sim 10 \mathrm{MW}$ radio frequency (RF) heating and current drive systems in next 2 years and $4 \mathrm{MW}$ neutral beam injection system in 3 4 years. It is currently a unique facility to explore some of critical issues relating to steady-state operation with the shaped plasma cross-section. It could become a good test bench, especially regarding the technology of steady-state diverter control and physics of long pulse operation with non-inductive current drive and experience achievement of full superconducting machine operation.

The institute of plasma physics, Chinese Academy of Sciences (IPP/CAS or ASIPP) has EAST as new project and simultaneous the HT-7 superconducting tokamak in operation. Significant progress has been achieved both in the EAST construction/operation and the HT-7 experiments with many contributions from broad international collaboration. In last few years, HT-7 experiments were strongly oriented to support the EAST project both physically and technically $[3,4]$. HT-7 is still a good machine for some of specific physical investigations, particularly, long pulse discharges, edge plasma turbulence, etc. Experience and experiment from the HT-7 operation become one important part of the EAST basis and certainly speeds up the EAST procedure.

This paper will report the main progress of machine modification and operation on EAST and some experiments on HT-7. It is organized as follows: Section 2 describes the development of systems on EAST, mainly, the new in-vessel structure. The experimental progress with the new in-vessel structure is given in section 3. Some of the experimental results in HT-7 are reported in section 4 followed by a summary and near future plan in section 5 .

\section{Development of systems on EAST}

The first EAST plasma was achieved under the full metal PFCs condition[2]. EAST as a full superconducting tokamak is aimed for long pulse (60-1000s) high performance operation, which requires specific in-vessel structures and PFCs. It should be capable to handle the particle and heat fluxes through the plasma boundary to the wall in a variety of operation scenarios under steady-state condition, as well as to protect vacuum vessel and other components such as magnetic sensors, internal cryo-pump etc from direct plasma interaction. The in-vessel structure is a complicated integration of multi-systems as shown in Fig.1. They include the fully actively water cooled PFCs and their supporting structures, a full set of magnetic inductive sensors for machine operation and plasma control, the divertor cryopump, the actively water cooled internal coils for vertical stabilization control, divertor probe arrays, baking system and thermal coupler etc. The system of the actively cooled PFC is a key element in construction of the new in-vessel structure. Fig. 2 shows the EAST in-vessel structure together with ICRF antenna and LHCD launcher after full construction. The geometry is designed as top-bottom symmetry to accommodate both double null or single null divertor configuration.

In the first stage of the machine operation, total heating and current drive power will be about $8 \sim 10 \mathrm{MW}$. But peak heat flux will not be more than $3.6 \mathrm{MW} / \mathrm{m}^{2}$ on the divertor plates from the B2-Eirene simulation based on typical EAST operation scenario. From economical and technical consideration, brazed tiles are not employed in the initial PFCs engineering instead of using bolted tiles. The PFCs consist of a plasma facing surface affixed to an actively cooled heat sink. All plasma facing surface are one kind of multi-element doped graphite materials[5]. 
The $15 \mathrm{~mm}$ or $20 \mathrm{~mm}$ (especially for divertor) thick graphite tiles are bolted to the copper alloy $(\mathrm{CuCrZr})$ heat sink and retrained through the spring washers that allow limited deformation during thermal expansion. This mechanically restrained structure is used for all PFC system. It is the thermal conductance across the tile to heat sink interface that are very important for the performance of a bolted tile. A thin piece $0.38 \mathrm{~mm}$ of graphite sheet is used between the tile and the heat sink to improve the thermal contact. Bolted structure should provide a minimum $0.2 \mathrm{MPa}$ average pressure over the contact area. Water-cooling channels are drilled holes directly along the $20 \mathrm{~mm}$ thick heat sink plates. Such a structure with $2 \mathrm{t} / \mathrm{h}$ water mass flow rate for inner target, outer target and dome can maintain plasma facing surface temperature around $800^{\circ} \mathrm{C}$ under peak heat load up to $3.6 \mathrm{MW} / \mathrm{m}^{2}$.

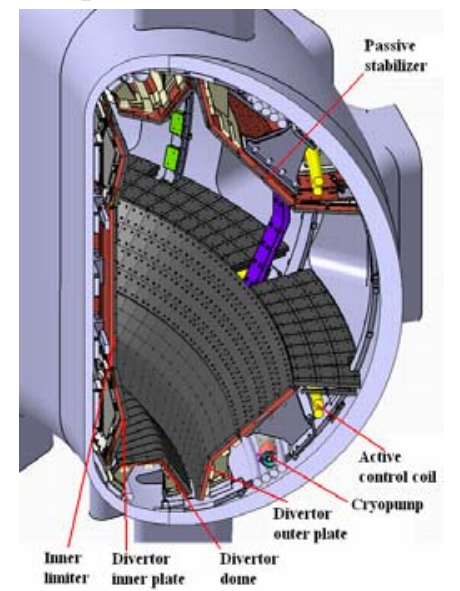

Fig.1 Elevation view of EAST in-vessel struture

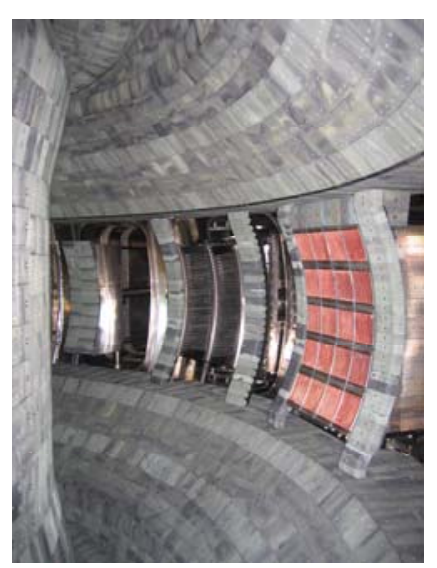

Fig.2 Picture of in-vessel together with the ICRF

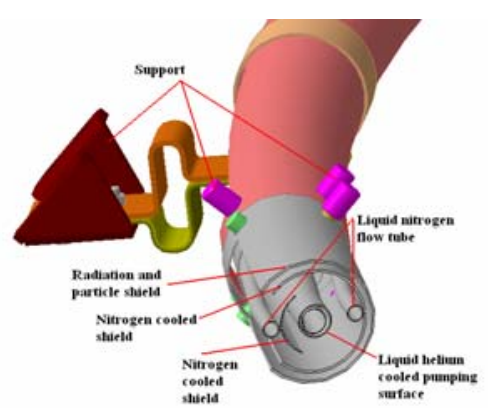

Fig.3 Elevation view of EAST toroidally continuous cryopump antenna and LHCD launcher

The vertical targets and dome form a "V" shape and expect particles remain in this region to help distribute heat load uniform on divertor plates. Two gaps between inner, outer target and dome were provided with total $180 \mathrm{~m} 3 / \mathrm{s}$ gas conductance for particle and impurity exhaust by cryopump. Other PFCs except the divertor region have the same structure but with lower cooling capability and allow maximum heat load $0.5 \mathrm{MW} / \mathrm{m}^{2}$. The passive stabilizer is placed on the outer radius of the plasma above and below midplane. The thickness of the stabilizer is $30 \mathrm{~mm}$ copper alloy with carbon tiles for protection and coolant channels for cooling. All PFCs were divided into 16 modules in toroidal direction, respectively. They can be taken in and out from vacuum vessel horizontal ports, and easy for maintaining and modifying. The PFCs modules have some flexibility relative to the vacuum vessel in order to reduce the magnitude of the differential thermal expansion loads that can occur both during operation and $350{ }^{\circ} \mathrm{C}$ baking conditions. Halo current considered $50 \%$ of plasma current and its toroidal asymmetry factor is 2 .

In-vessel coils and cryopump are installed behind first wall and divertor plates to avoid facing plasma directly and fixed to vacuum vessel wall. The divertor design features in-vessel cryopump located in a plenum under the outer divertor plate and passive stabilizer, which is near the gap between the doom target and outer target. It provides pumping speed $>15 \mathrm{~m}^{3} / \mathrm{s}$. The pump is continuous tubes with slot open to vacuum vessel wall and are supported by flexible supports (Fig.3). Other components including the 4 DC glow discharge anodes and 2 RF conditioning antenna were also installed for wall conditioning. The thermal couplers are 
embed in the graphite titles of the liner, limiter and diverter plates for machine operation and wall conditioning. Two movable molybdenum limiters have been installed, which allow radial movement from $2.26 \mathrm{~m}$ to $2.42 \mathrm{~m}$.

Magnetic measurements consist of: 3 Rogowiski coils in vacuum chamber for plasma current and two outside the vessel including the vessel current, $2 * 37$ flux loops, $3 * 39$ probe arrays, 2 Mirrov coil arrays along poloidal direction and a 20 coil array in toroidal direction, 2 sets of diamagnetic and compensation coils, 8 Rogowiski coils surround the support legs for halo current, 4 sets of poloidal saddle coils arrays sysmetric located at 4 toroidal positions. These magnetic sensors provide sufficient information for machine operation, plasma control and physics analysis. Two Langmuir probe and mach probe arrays were installed in top and bottom divertor plates and dome respectively. There is a single channel laser interferometer with the vertical sight line at $\mathrm{R}=1.9 \mathrm{~m}$, which provide a line integrated density and is used also for density feedback control. Two visible CCD camera look at plasma in tangential sight line to monitor plasma discharges. Additional 20 diagnostics installed presently can provide measurements of electron/ion temperatures, surface temperature of the liner or diverter plates, radiation power, and information of soft- $\mathrm{X}$ ray, visible to near UV radiation of impurities, $\mathrm{H}_{\alpha}$ radiation etc. Hard-X ray and neutron flux measurements are also available for LHCD experiments and monitoring the runaway electrons.

Plasma discharges are controlled by a plasma control system (PCS) built in collaboration with GA, which is similar to the PCS of DIII-D, but with new EAST features such as the coil current ramping rate limitations due to the eddy current heating on the superconducting cables, IC power supply command algorithms, etc. The RF systems at ICRF with $1.5 \mathrm{MW}$, $30 \sim 110 \mathrm{MHZ}$ and at LHF with $2 \mathrm{MW}, 2.45 \mathrm{GHz}$ are available for heating and current drive experiments as well as wall conditioning and discharge pre-ionization.

\section{The first experiments with new PFCs}

EAST as a full superconducting tokamak has new features compared to conventional tokamak and also those tokamaks only with the toroidal superconducting coils. These issues, particularly, the limitation of current ramping rate in PF coils, relative weak coupling between plasma and PF coils, PF field penetration through the vacuum vessel and thermal shielding into the plasma etc affecting the machine operation have been discussed elsewhere[2]. The graphite wall with the vacuum chamber was baked up to nearly $300^{\circ} \mathrm{C}$ and $200^{\circ} \mathrm{C}$ continuous for about 10 days before the plasma discharges. However burn through mainly caused by out-gassing from the graphite titles caused difficulty for the further current ramping up after break down. To achieve reliable break down and plasma current ramping up, the machine was boronized by RF discharge, which was used as routine wall conditioning in HT-7. The working gas was hydrogen in 2007 and then switched to deuterium for recently campaign.

The experiments have firstly performed using pre-programming shape control (elongation and diverter) and feedback control for plasma position and current with the (copper) internal control coils (ICs) for vertical stabilization. The principal goal of this experiment was achieved with the appearance of stably controlled diverted plasmas with sufficient elongations and triangualarities as shown in Fig.4. To verify the shaping capabilities of the poloidal field system and vertical stabilization of ICs, highly shaped plasma at various configurations has been stably produced shown in Fig.4 and Fig.5. The full equilibrium reconstruction both by 
EFIT(GA) and IPPEQ(ASIPP) identified the following configurations: double null configuration with kappa $=1.9$ and delta $=0.50$; top or bottom single null configurations with kappa $=1.7$ and delta $=0.64$, which almost covers all designed configuration in EAST. The discharges with plasma current ranged from 0.2 0.6MA, toroidal magnetic field from $2 \sim 3 \mathrm{~T}$ show the confinement consistent with Neo-Alcator scaling for ohmic plasmas. Such experiments provide the basis for algorithm development and optimization of real time control of plasma shape.

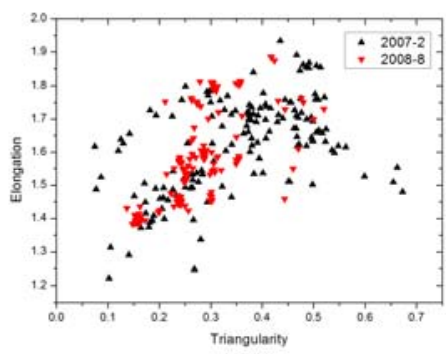

Fig.4 Operation space for highly shaped plasma discharges

\section{Fig.5 Oper
stability}

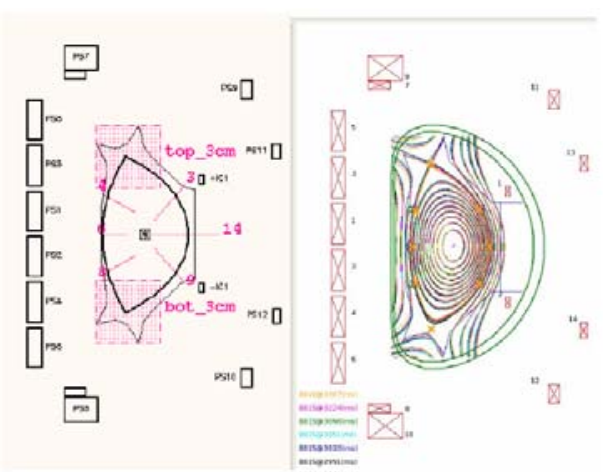

Fig.7 Left: reference point for iso-flux control, right: plasma shape controlled by iso-flux from $3 \mathrm{~s}$
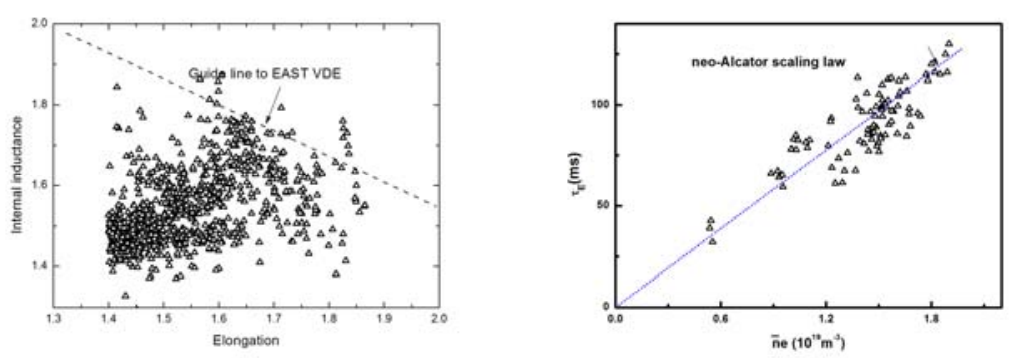

Fig.6 confinement scaling for ohmic plasma discharges

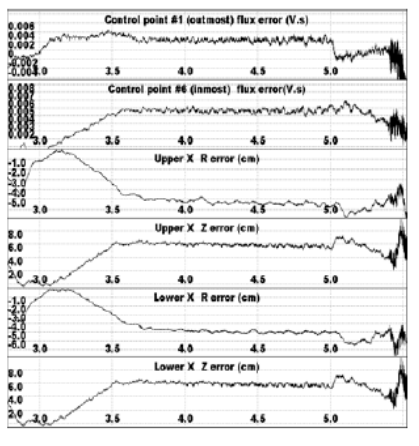

Fig.8 main controlled parameters at the isoflux control algorithm

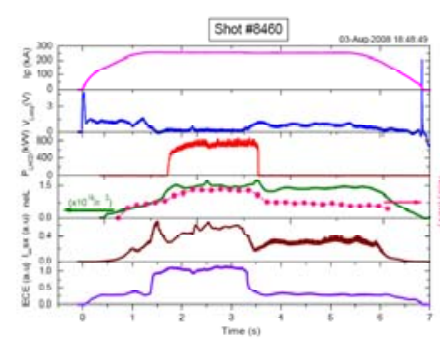

Advanced steady-state tokamak plasma operation relies on the exact control of the plasma shape in order to get better coupling to the heating RF waves, protect divertor by exact control of the striking points. The full reconstruction of the equilibrium has be performed by using efit $^{[6]}$ code routinely between shots. This kind of reconstruction was made to be real-time (RTEFIT) and sufficiently fast for the real-time shape control in DIII-D by using a fast loop and a slow loop calculations on separate CPUs. While RTEFIT has been done at a control cycle, the control reference points was determined at first. The flux difference between measured and pre-defined at the reference points were be controlled to be zero based on the called RTEFIT/ISOFLUX algorithm, which was firstly realized in DIII-D[7]. Under the collaboration with DIII-D, EAST also adapted DIII-D plasma control software system[8]. RTEFIT/ISOFLUX was primarily realized on EAST in 2008 summer campaign shown in Fig.7.

The control points were shown in cross symbol together with the plasma shape and flux surfaces as shown at the right of figure 7 . The upmost and lowest points are the upper and 
lower X points, respectively. The other points in the figure were chosen in such a way to mainly control the inner gap by the inmost point, outer gap by the outmost point, the upper and lower triangularities by the other points. Figure 8 shows the main controlled parameters at the isoflux control algorithm. It can be seen that the flux errors were well controlled below 5 mill-Vs for most of the discharge time under shape control which started from $3 \mathrm{~s}$. The $\mathrm{X}$ point position control is rather good for up X-point, but in the range of several centimeters for lower X-point. In order for X positions to be well controlled with much lower error, the shape control has to be sufficiently consistent with the fast vertical position control. This leaves to be fulfilled at the next experimental campaign.

The RF powers were applied in several aspects. The LHW was used for current drive both in sustaining plasma discharges and assisting the plasma start-up. The ICRF was mainly applied for pre-ionization for reliable plasma start-up and wall conditioning. Most of these experiments were performed by pre-programming control of plasma shape and feedback control of RZIp. The plasma shape and position were optimized to maximize the wave coupling into the plasma. Nearly $0.8 \mathrm{MW}$ LHW has been successfully delivered, from what about $0.65 \mathrm{MW}$ power has been coupled into the plasma shown in Fig.9. This power can almost sustain a fully non-inductive plasma discharge at $\mathrm{Ip}=250 \mathrm{kA}$ and line averaged density of $\sim 1.5^{*} 10^{19} \mathrm{~m}^{-3}$. The current driving efficiency estimated from power scanning under this condition is about $0.8^{*} 10^{19} \mathrm{Am}^{-2} \mathrm{~W}^{-1}$ shown in Fig.10. It is double higher than the LHCD efficiency obtained in HT-7, but smaller than that in tore-supra mainly due to the lower volume averaged electron temperature in present experiments. The plasma discharges can be sustained over 20 seconds in such operation scenarios (Fig.11), which were mainly limited by unstable coupling due to pre-programming shape control and the power supply stability of klystrons inside the LHCD system.

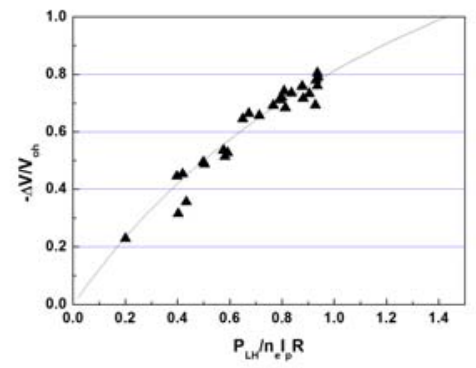

Fig.10 Normalized LHCD power vs variation of surface voltage

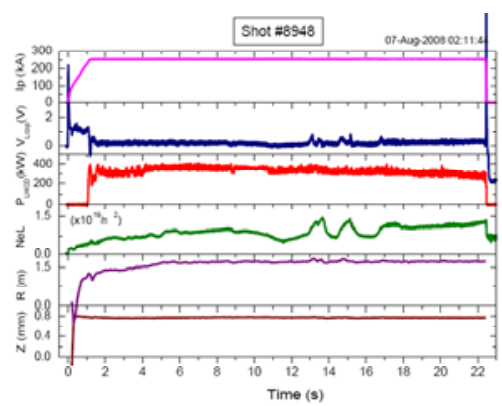

Fig.11 Long pulse discharge sustained by LHCD for 22s

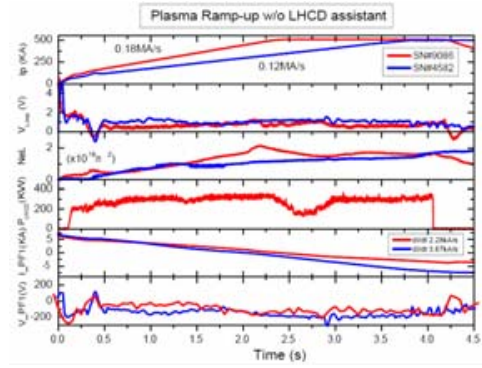

Fig.12 Plasma current ramp-up with/without LHCD.

The new machine shows its unique features during experiments, which can address a number of important issues for ITER operation, such as plasma initiation, ramp up and control with constraints of superconducting coils. Very low plasma ramp rate of $0.12 \mathrm{MA} / \mathrm{s}$ during start up phase have been obtained under a very well boronized wall condition. Such low ramping rate led to operation of the PF coils and power supplies in the region far from their limits. However, plasma current ramping with such low rate couldn't be always reliable due to burn through at low temperature. LHCD applied at plasma start up phase can significantly reduce the current ramping rate in PF coils or voltage applied at PF coils for the same plasma current ramping rate. This operation mode, on one hand, minimized the heat deposition on the PF coils caused by AC losses, and hence, increases safety of machine operation. On another hand, 
it allows better plasma control, particularly, during shaping phase due to the larger voltage regulation margin of PF power supply. Typical results with and without LHCD start up assistants are compared in Fig. 12.

In instance of frequent variation of the plasma performance such as large ELM burst, monster sawteeth, etc, the control demands quick response of the PF currents to maintain the plasma equilibrium and keep the configuration. The continuous heat deposition on the coils can limit the pulse length of the discharge when the magnet temperature rises to the marginal value. Therefore, effects of plasma discharge and operation scenarios on the superconducting magnets, particularly on poloidal magnetic system and cryogenic system, are constrains for future steady-state machine operation. These issues have been investigated by simulating discharges or during plasma discharges. Plasma disruption seems not be a direct safety constrain for the superconducting PF coils due to the strong shielding effect from the vacuum vessel. But such algorithm has to be adopted in controlling the PF current ramping down rate after termination of the plasma discharge to avoid higher thermal load on the magnets and cryogenic system. The energy deposition on TF coils, TF cases and PF coils due to the AC losses caused by varying PF currents has been investigated at different current ramping rates for duration up to 620s. Figure 13 the outlet temperature rise of the CS during the excitation at constant rate of $1 \mathrm{kA} / \mathrm{s}$. The temperature rise seems to be saturated for sufficient excitation duration and is still below the safety margin of $1 \mathrm{~K}$. The results showed that the capability of the cryogenic system is sufficient to keep the magnet temperature and coolant helium pressure much lower than the marginal values if the current varying rates in PF coils do not exceed the designed specification. The details for the safety operation for the superconducting magnets will be discussed elsewhere [9].

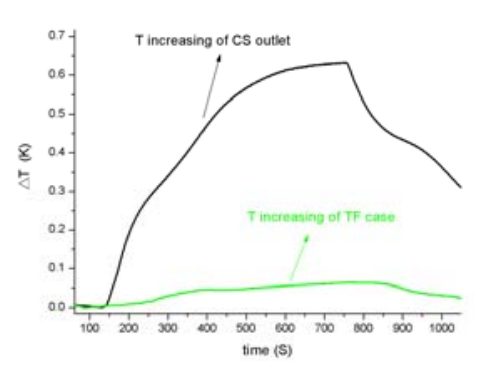

Fig. 13 Outlet temperature rise of CS at exciation rate of $1 \mathrm{kA} / \mathrm{s}$

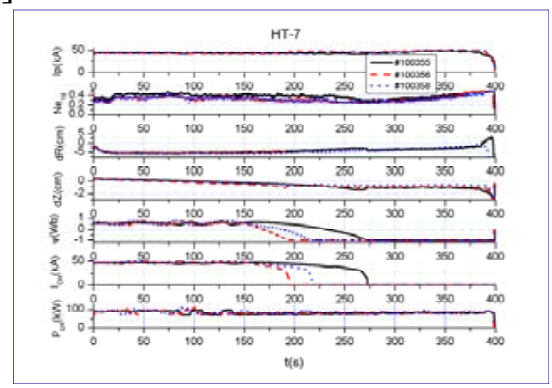

Fig.14 Repeatable long pulse discharges for 400s

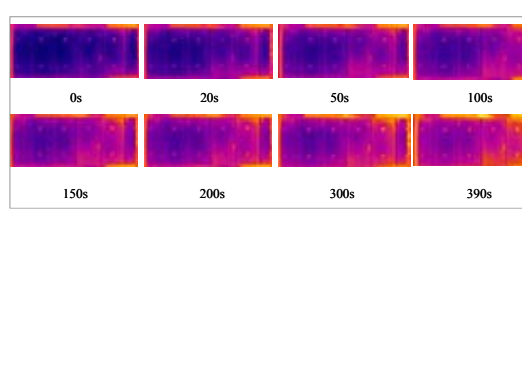

Fig.15 Surface temperature of belt limiter in the high field side.

\section{Experiments in HT-7}

Since the last IAEA meeting, experiments in the HT-7 tokamak focused on long pulse discharges under different scenarios and high power heating to support EAST experiments both physically and technically. To meet the long pulse operation requirements, several systems around HT-7 were modified. The poloidal control algorithm was developed based on real time magnetic equilibrium reconstruction with the improved magnetic diagnostics. New heat sink technology and material were adopted to replace the belt limiter at high field side, which validates and supports construction of the EAST in-vessel components.

The long pulse experiment was performed by driving the plasma current fully non-inductively through the use of LHCD, which was realized in two different scenarios. The first one is via 
feedback control of the magnetic swing flux of the transformer at a constant. The second way is so-called transformer-less discharges, which was realized by over current drive up to reversed saturation of the transformer and then switch off the current in the central solenoid. Experimental observation indicates that the photon temperature and cut-off energy of supra thermal electrons are smaller in the transformer-less discharges than in the first scenarios. These techniques has been used successfully to sustain the plasma discharges for 400s at Ip $50 \mathrm{kA}$ shown in Fig 14, which is the new record in HT-7. The experiments indicate the success of the new built belt limiter and also suggests important role of supra thermal electrons on the heat load at the limiter as shown in Fig. 15.

The operation scenario, which combines LHW and IBW, was optimized to achieve high current drive efficient and high plasma performance for long pulse operation. The current drive efficient with the combination of two waves was investigated and was $10 \sim 20 \%$ higher than LHCD only. Both duration of discharges and injected current drive and heating power in this scenario have been greatly extended to $10 \mathrm{~s}$ and $1.5 \mathrm{MW}$ compared to previous experiments of $8 \mathrm{~s}$ and 1.0MW respectively.

Sawtooth oscillation is one of the most important MHD instabilities in tokamaks. Recently, progress has been achieved by newly developed 2D ECEI and high-resolution soft-x-ray multi-arrays to verify new models[10,11]. There are developed 5 high-resolution soft-x-ray arrays and a 2D ECEI system 8(radial)x16(vertical) channels on HT-7 for investigation of sawtooth activities and temperature turbulence. Resolution of ECEI for each pixel is about $1 \mathrm{~cm}$ (radial) $\times 1.1 \mathrm{~cm}$ (vertical). The time resolution is about 4 microseconds and the system noise to signal level is about $1 \%$. The high-resolution soft-x-ray arrays locate in one poloidal cross section with different poloidal view angles, and each array have 46 channels, covering the whole plasma region.

Islands coalescence is observed before reconnection during $m=1$ sawtooth crash by the $2 \mathrm{D}$ ECEI system. The experiments are under pure ohmic discharge with toroidal magnetic field $\mathrm{Bt}=1.9 \mathrm{~T}$, plasma current $\mathrm{Ip}=170 \mathrm{kA}$, central line-averaged density of $2.4 \times 10^{19} / \mathrm{m}^{3}$ and edge safety factor qa 3.3. The poloidal beta is about $\beta_{\mathrm{p}} \sim 0.16$.

A representative view of the sequence of $2 \mathrm{D}$ ECEI images during the reconnection process is provided in Fig 16. In the top of the figure, a time history of the temperature fluctuation measured by one of the 128 channels (inter) illustrates the typical precursor oscillations and crash procession. The reconnection happened on the $\mathrm{q}=1$ radius in the LFS makes the magnetic field line open. As a result, the heat flow escape to the outside of the inversion radius collectively. Similarly, magnetic reconnection is also found by 2D image in the HFS. The character of magnetic reconnection near inversion radius during sawtooth oscillation is analogous to that observed on TEXTOR [10].

Fig. 17 shows the evolution of $\mathrm{m}=2$ islands coalescence in $\mathrm{q} \sim 1$ surface during precursor phase of sawtooth. The first island rotates anticlockwise passing through the diagnostic region in the frame 1 and 2. The island still stays above the diagnostic region for some unknown reasons in the subsequent frame 3, while simultaneously another island rotates into the diagnostic region below. The two islands reconnect near the mid-plane in only about 10 microseconds as typically shown during the frame 3 and 4 and finally, the two islands coalesce into one island as $m=1$ mode in the frame 5 . After the formation of the $m=1$ island, the heat in the core escape to the outside of the inversion radius during sawtooth crash during the frame 6 to 8 . 

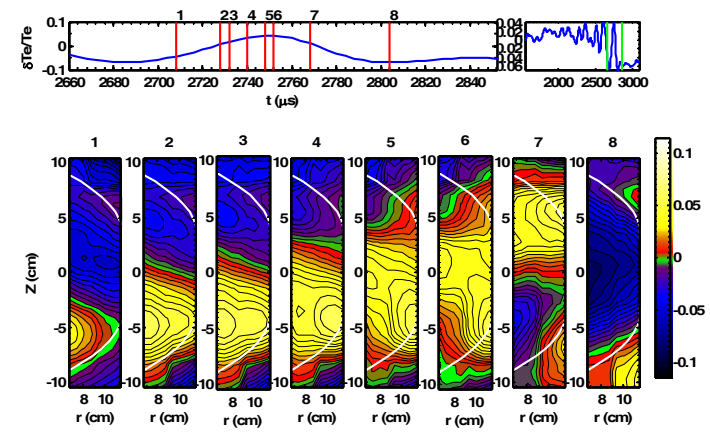

Fig 16. The 2D image of temperature fluctuation evolution during the reconnection process (Low field side). The inversion radius shown as the white line is determined from the assumption that fluctuation is most quiet near the inversion radius.(\#93513, about 537ms)

The ECEI presents clearly the charateristics of the temperature fluctuation during the sawtooth crash phase. However, the observation is limited by a relatively small spatial region. Tomography of high-resolution soft-x-ray arrays can give the sawtooth crash structure. The Fourier-Bessel inversion method is used in the reconstruction by choosing $\mathrm{M}=2-3$ and $\mathrm{L}=6-8$ [11]. In order to present a clear picture of the crash, the sawtooth activity in Lower Hybrid Current Driven (LHCD) plasma is analyzed due to the large fluctuating amplitude (20-30\% of the total emission in the central sightline) for distinguishing the crash and precursor stage. In order to obtain the picture of variation of the plasma pressure (hence heat flow) due to sawtooth oscillations, Singular Value Decomposition (SVD) technique is employed to extract the perturbative components from the total signals. The reconstruction of the mode structure with perturbation signals, which reflects the variations of the plasma pressure caused by the oscillations in plasmas, is more reliable.

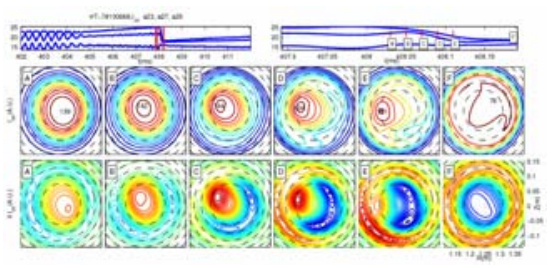

Fig.18. Reconstructed sawtooth crash picture by topography. The top row shows the evolution of the soft- $x$-ray signals. The middle row is topography from total signals, and the bottom row is the contour plot of the reconstructed perturbation signals extracted by SVD method.

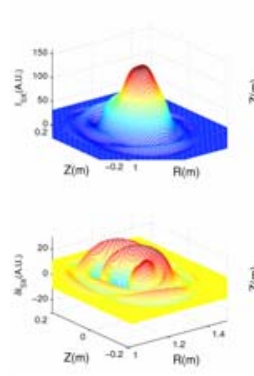

Fig.19. soft-x-ray profile during sawtooth crash by topography. The top frames are reconsturcted from total signals and the bottom frames from perturbative signals.

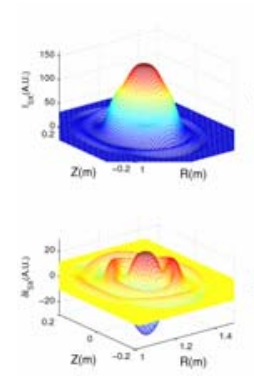

Fig. 20. soft- $x$-ray profile during sawtooth crash by topography. The heat flow transfer across both the two $x$ points of the $m=2$ island can be identified in the bottom frame. 
By using this method, the reconstructed sawtooth crash process from perturbation signals of a sawtooth crash is shown in the bottom frames in Fig. 18. It shows that, a large heat flow transfer across the $\mathrm{x}$ point of (both the $\mathrm{m}=1$ and $\mathrm{m}=2$ ) islands $(\mathrm{C}-\mathrm{E})$ and then the transferred energy gradually spreads poloidally over the peripheral region near the $q=1$ surface $(E-F)$, while the reconstructed sawtooth crash process from total signals, as shown in the middle frames in Fig. 18, seems to be consistent with the Kadomtsev's model, which was also concluded in Ref [11]. The asymmetric heat flow can occur both in low field side and high field side, which is different from the observations in high $\beta$ plasmas on TFTR [12]. The heat flow characteristics near the $\mathrm{x}$ point during sawtooth crash phase are consistent with the observations by 2D ECEI. Another type of sawtooth crash is shown in Fig. 19, where the heat flow transferring is not across the $\mathrm{x}$ point of the $\mathrm{m}=1$ island instead of one of the $\mathrm{x}$ points of the $\mathrm{m}=2$ magnetic islands (this mode is rather weak but can be identified). This suggests that the large $\mathrm{m}=1$ component in the soft-x-ray signals is due to the large asymmetric heat flow during sawtooth crash phase rather than the abruptly increasing of the $m=1$ magnetic island. Another interesting result is shown in Fig. 20. The heat flow transfer across both the two $x$ points of the $\mathrm{m}=2$ magnetic islands, with a little asymmetry in low field side and high field side. In this example, the $\mathrm{x}$ point of the $\mathrm{m}=1$ islands is overlapped with one of the o point of the $m=2$ magnetic islands, which can be identified by the asymmetry of the two o points of the $\mathrm{m}=2$ magnetic islands. This result again demonstrates that the sawtooth crash is not caused by the fast reconnection of the $\mathrm{m}=1$ magnetic island.

The observation above strongly suggests that the sawtooth crash is not caused by the fast reconnection of the $\mathrm{m}=1$ magnetic island, while the picture is in agreement with the turbulence model[13]. For turbulence model, the large heat flow and hence the crash is the result of the fast energy and particle diffusion in the magnetic stochastic region around the separatrix of the magnetic islands.

Electron mode and ion mode coexist in high density ohmic plasma $\bar{n}_{e} \geq 4.5 \times 10^{19} \mathrm{~m}^{-3}$ has been observed by 2D ECEI. The local wavenumber-frequency spectrum by two-points correlation technique are shown in Fig 21. The two modes are found coexist at $\mathrm{r} \sim 10 \mathrm{~cm}$ in the low field side as well as at $\mathrm{r} \sim 13 \mathrm{~cm}$ in the high field side (HFS). Ion temperature gradient mode in density fluctuation was observed by far-infrared (FIR) scattering and beam emission spectrum (BES), but has not been reported in the temperature fluctuation yet.

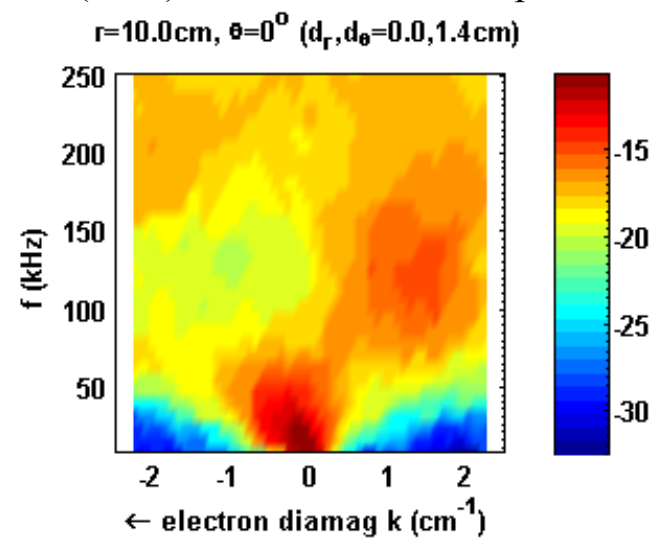

Fig.21 (a) LFS (\#93532,93533)

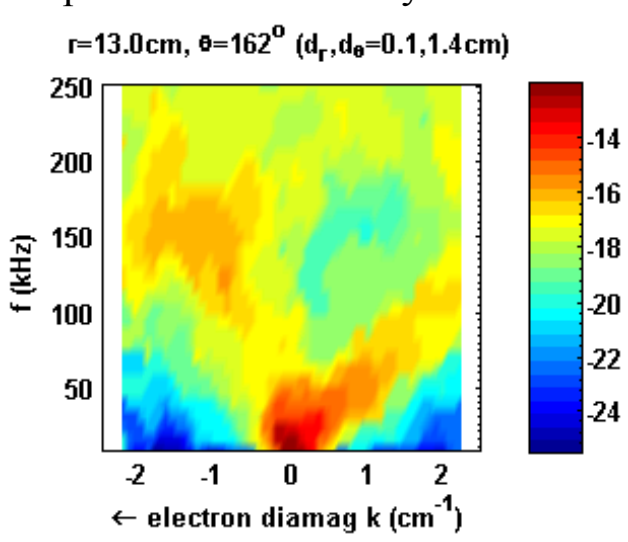

(b) HFS (\#93572,93573)

Since there are two windows located at the top of poloidal cross sections separated 120 degrees toroidally for the Langmuir probes, the long-distance correlational potential structure could be detected in poloidal and toroidal directions. The probe system in one window 
consists of two arrays at a distance of $2 \mathrm{~cm}$. Another probe is a tri-probe measuring the local floating potential, electron temperature and density simultaneously. The experiments were carried out in high $\mathrm{q}_{\mathrm{a}}(6.6 \sim 8.5)$ plasmas with line averaged density of $1 \sim 2 \times 10^{19} / \mathrm{m}^{3}$. The poloidal mode numbers and the toroidal mode numbers from the floating potential fluctuations have been calculated, as shown in Fig.22. The central frequencies of the GAM of the two shots are both $12 \mathrm{kHz}$. The mode numbers from $1 \mathrm{kHz}$ to $12 \mathrm{kHz}$ are all below one.

The radial wavenumber-frequency spectrum $S\left(k_{r}, f\right)$ of the GAM could be estimated by the step structure of the Langmuir probes, shown in Fig.23 from $1 \mathrm{kHz}$ to $12 \mathrm{kHz}$. The spectral averaged wavenumber and spectral width are respectively $\bar{k}_{r}=0.83 \mathrm{~cm}^{-1}$ and $\Delta k_{r}=4.6 \mathrm{~cm}^{-1}$, which corresponds to $k_{\mathrm{r}} \rho_{i} \sim 0.065$ and $\Delta\left(k_{\mathrm{r}} \rho_{i}\right) \sim 0.31$ (The positive direction of $\mathrm{k}_{\mathrm{r}}$ is outward). This is in the range for the GAM expected by theory [14] and simulations[15] and consistence with the experimental results in other devices[16].

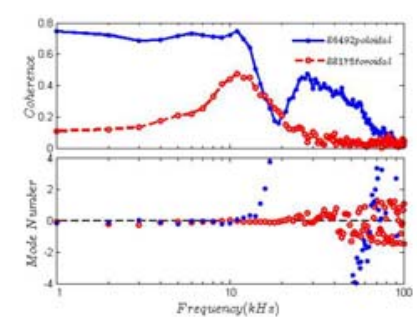

Fig.22. Coherent coefficients and mode numbers between two floating potentials separated poloidally and toroidally

The squared self-bicoherence is calculated, as shown in Fig.24. Only the first quadrant is plotted because of the symmetry. The value of the tri-wave group $\left(f_{1}, f_{\mathrm{GAM}}, \mathrm{f}_{1}+\mathrm{f}_{\mathrm{GAM}}\right)$ is higher than others, indicating the nonlinear three wave coupling exists at the GAM frequency. This is similar to other experimental results except the values are somewhat smaller, which may be caused by the turbulence situation in this low discharge current.

\section{Summary and near future plan}

Since last IAEA meeting, significant progress in construction of the fully actively water-cooled in-vessel components and control in obtaining highly shaped plasma has been made on EAST. The primary achievements, particularly, the experiences from last two years provide us confidence that the highly shaped plasma with relevant performance could be sustained by RF powers for long duration, although significant improvements are needed for reliable machine operation and effective experiments.

Presently, a $2 \mathrm{MW}$ lower hybrid current drive system at $2.45 \mathrm{GHz}$ and a $1.5 \mathrm{MW}$ ICRF system at $30-110 \mathrm{MHz}$ are in operation. A new $4.5 \mathrm{MW}$ ICRF system at $25-70 \mathrm{MHz}$ will be available in 2009. The present LHCD system is planed to be upgraded to 4MW, where existing klystron of $100 \mathrm{~kW}$ will be replaced by new klystron of $200 \mathrm{~kW}$. Capability of corresponding power supplies and water cooling etc will be expanded within next 2 years. The total heating and current drive power will be 10MW before end of 2010. This power is much higher than the $\mathrm{H}$-mode threshold, which is about 4MW for standard EAST operation scenario at 1MA plasma current and toroidal field strength of 3.5T. The flexibilities of heating scenarios and current drive in controlling current density profile provide the possibilities to operate EAST in high performance regime with edge and/or internal transport barrier. A new proposal for a $4 \mathrm{MW}$ LHCD system at $4.6 \mathrm{GHz}$ and a $2 \sim 4 \mathrm{MW}$ neutral beam injection system at $50 \sim 80 \mathrm{keV}$ 
will be funded for next 4 years.

In the next two years, diagnostics on EAST will provide measurements of all key profiles, which include Thomoson scattering for density and electron temperature, charge exchange recombination spectroscopy based on a diagnostic beam for ion temperature and rotation, bolometry for radiation power, X-ray crystal spectroscopy for ion/electron temperature, multi-channel ECE or 2D ECE image for electron temperature and fluctuation, visible bremstahlung for effective charge number, multi-channel DCN laser interferometer for density, HX-ray arrays for LHCD, neutral partical analyzer for energetic particles, optical and spectroscopic diagnostics for impurity, etc. Some of them will be built via international collaboration. These diagnostics should be sufficient to describe the basic plasma performance and for integrated modeling and data analysis.

HT-7 will be still operated before EAST is equipped with sufficient heating and current drive power and diagnostics. Its experiments will focus on issues related with the plasma and wall interaction under long pulse condition and some of specific topics, such as, MHD instabilities, transport and turbulence by Langmuir probes, newly developed $\mathrm{CO} 2$ laser scattering and 2D ECE image systems.

\section{Acknowledgements}

This work was supported by the National Natural Science Foundation of China under Grant No. 10725523 and No. 10721505 and partially by the Core-University Program of Japanese Society of Promote Sciences.

\section{Reference}

[1] Yuanxi Wan, et al., "Overview progress and future plan of EAST Project" OV/1-1, 21th IAEA-FEC in Chengdu, Oct. 2006.

[2] Baonian Wan, et al., "First plasma in EAST" PD/P6-5, ibid

[3] Baonian Wan, et al., Nucl. Fusion 43, (2003) 1279.

[4] Baonian Wan, et al., Nucl. Fusion 45, (2005) S132.

[5] Baonian Wan, et al., Journal of Nuclear Materials Vol. 313-316 2003 p. 127-134

[6] Lao, L.L., et al., Nucl. Fusion 25 (1985) 1611

[7] J.R. Ferron et al., Nucl. Fusion, 38, No. 7 (1998)

[8] B.J. Xiao, et al., "EAST plasma control system", FUSION ENGINEERING AND DESIGN (2008) $83-181$

[9] Yuanxi Wan, et al., "Risk Analyses and Optimization of Safe Operation for Advanced Superconducting Tokamak" FT/P3-24, this conference

[10] Park H. K. et al., Phys. Rev. Lett. 96, (2006) 195004

[11] Janichi C. et al, Phys. Rev. Lett. 62 (1989) 3038

[12] Nagayama Y. et al, Phys. Plasmas 3, (1996) 1647

[13]Lichtenberg A. J., Nucl. Fusion 24, (1984) 1277

[14]P. H. Diamond et al., Plasma Phys. Control. Fusion 47, (2005) R35

[15] T. S. Hahm et al., Plasma Phys. Control. Fusion 42, (2000) A205

[16]A. Fujisawa et al., Nucl. Fusion 47, (2007) S718 